\title{
VISUALISATION TOOLS FOR DESIGN OF MARITIME SURVEILLANCE SYSTEM
}

\author{
Bojan Džolić, 2, *, \\ Nikola Tošiće, \\ Vladimir Orlić, \\ Mladen Veinović ${ }^{1}$
}

\author{
'Singidunum University, \\ Belgrade, Serbia \\ 2Vlatacom Institute, \\ Belgrade, Serbia \\ ${ }^{3}$ Faculty of Transport and Traffic Engineering, \\ University of Belgrade, \\ Belgrade, Serbia
}

\begin{abstract}
:
The aim of this paper is to describe the application of Matlab for the design of the sensor network within a real-world Maritime Surveillance System. In the phase of planning and solution preparation of new Maritime Surveillance System project, it is necessary to analyse the zones of monitoring, sensors arrangement, and spatial distribution in order to make the best decision. With a goal to define the observation zone of interest, a software solution is made in Matlab using built-in functions for location computing in World Geodetic System, a graphical representation in Google Earth and a library that supports working with Google Earth formats for export. The sensors supported in this Maritime Surveillance System are Electro-Optical systems - multi-sensor cameras, X-band and Ku-band microwave radars and High Frequency Over The Horizon Radars. With this software solution, a particular sensor's observation zones, along with global coverage zone are determined at the real location. Coverage diagrams are plotted as Google Earth objects, generating a KML export file. In this way, easy manipulation over potential solutions is provided, with high flexibility and portability, leading to a more detailed assessment and decision making in Maritime Surveillance System design.
\end{abstract}

Keywords:

Maritime Surveillance System, High Frequency Over The Horizon Radar, Electro-Optical systems, MW radars, KML.

\section{INTRODUCTION}

Nowadays it becomes clear that control of territorial waters is not enough to ensure secure flow of goods from Exclusive Economic Zone (EEZ) [1]. More and more efforts are being made to protect the complete EEZ: a sea area stretched from territorial waters up to $200 \mathrm{NM}$ in direction of open sea in which countries have exclusive rights regarding the exploration and use of marine resources of the sea. Controlling this zone represents technological, financial and organizational challenge [2]. In order to achieve better coverage of such a large area the observation zone is divided into several smaller subzones. Critical areas are ports, oil platforms, territorial waters and EEZ. According to the functionality of the sensors themselves for each of the above mentioned zones, a different type of sensor is used. The sensors typically supported in this Maritime Surveillance System are Electro-Optical systems - multi-sensor
Correspondence:

Bojan Džolić

e-mail:

bojan.dzolic@vlatacom.com 
cameras (EO), X-band and Ku-band microwave radars and High Frequency Over The Horizon Radars (HFOTHR). The data collected from all sensors are integrated into a unique software platform. From that software platform the operators are able to monitor and track all vessels detected from the all above mentioned sensors. It is very important that integration of the sensors does not deteriorate the continuity of tracking ships.

In the first phase of MSS design, it is necessary to determine the positions of the sensors themselves in relation to the client's requirements and to make the coverage areas of all the sensors individually. The main goal is to define complete observation zone of interest using a software tool. With these software solutions, a particular sensor's observation zones, along with global coverage zone are determined at the real location. In this way, easy manipulation over potential sites is provided, with high flexibility and portability. After this phase of MSS design the field survey is coming in order to find the most favorable location for the installation of sensors. Automated approach in design phase of MSS based on HF-OTHR technology is of crucial importance, since these systems are very complex and sophisticated resolving of practical issues is more than necessary ([3] - [7]).

\section{MARITIME SURVEILLANCE SYSTEM CONCEPT}

The main goal of MSS is to support a large number of sensors in order to achieve effective coverage of zones of different sizes: from local zones (ports, corridors) to the complete EEZ - which logically mean a multitude of sensors.

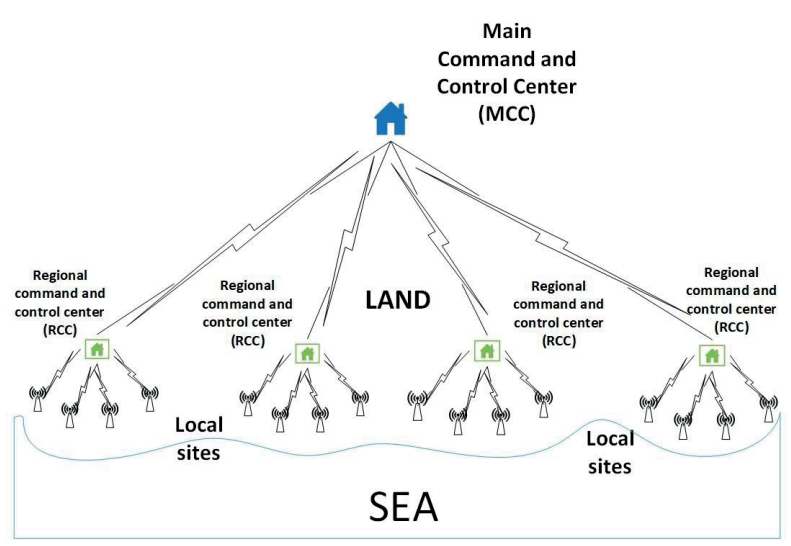

Fig. 1. Maritime Surveillance System concept
The solution is based on integration of several types of sensors into unique surveillance network that includes one main control centres (MCC) and several regional control centres (RCC), as shown on Fig. 1.

All sensors are divided in regions with Regional Command and Control centers - RCC and all RCC's are integrated in national network with the Main Command and Control center - MCC. In this way, the territorial hierarchy is achieved from the MCC down to the local site level.

In the phase of planning and solution preparation of new Maritime Surveillance System project, it is necessary to analyse the zones of monitoring, sensors arrangement, and spatial distribution in order to make the best decision. Each sensor has its own defined coverage zone, depending on type and particular model.

The following sensors are supported within our solution:

- Vlatacom Over The Horizon Radar - vHFOTHR,

- Maritime MW X band radars - SEAFALCON,

- Electro Optical multi-sensor cameras - vMSIS3CHD-C1200.

A MW radar type SEAFALCON [8] is a sensor with very high resolution and because of that feature it can detest very small objects, Fig. 2. Disadvantage of this radar is because this is the line of sight radar and needs to have optical visibility with the target. Because of the curve of the earth this sensor has huge limitation according to the coverage range [9], e.g. if MW radar antenna is installed on the standard telecommunication tower with the height of $50 \mathrm{~m}$ above the see level the maximum detection range should be around $45 \mathrm{~km}$. The area that needs to be reserved for installation of the tower, generators and equipment shelter is around (30 $\mathrm{x} 30) \mathrm{m}$.

In order to achieve coverage of complete coast line the radar network of MW radars should be used.

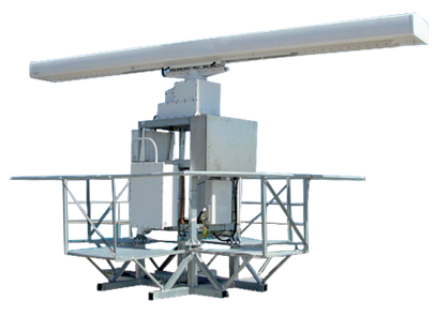

Fig. 2. MW X-band radar - GEM LD 2325 RK, [8] 
The vMSIS is a state of the art monitoring and surveillance system that integrates various high definition imaging sensors and provides long range target detection, recognition and identification based on superior sensors, optics and image processing [10]. System comprises a cooled MWIR high definition thermal imager, a color low light day/night high definition imager, and an optional SWIR imager, each of them with long range optics and a system hybrid real-time image stabilization, Fig. 3 .

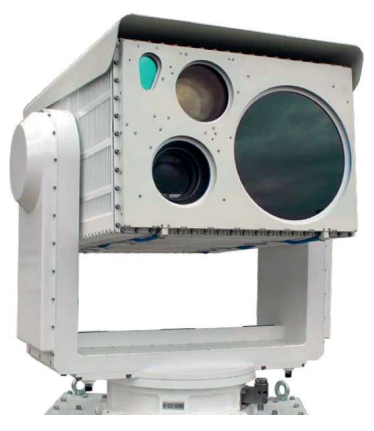

Fig. 3. EO camera - vMSIS3-CHD-C1200, [10]

The vMSIS camera is installed together with MW radar on a shared tower. The cameras are fully synchronized with MW radars and once the MW radar detects the object located on the see the camera will visualize that object displaying it on the screen located in MCC. Maximum coverage range for the camera is around 20 $\mathrm{km}$ in diameter.

The HF-OTHR is based on surface wave propagation, provides a unique capability to detect targets far beyond the conventional microwave radar coverage [2]. HF-OTHR radars use the frequency band of $3-30 \mathrm{MHz}$ to provide a large coverage that could extend up to 200 $\mathrm{NM}$ in range [11]. It has the ability to scan the area in angle width of $\pm 60^{\circ}$. A typical configuration is given in Fig. 4 . The radar consists of 2 subsystems: a transmitting (Tx) antenna array, receiving ( $\mathrm{Rx})$ antenna array and transceiver. Dimension of the sites directly depends on working frequency [12][13], e.g. if working frequency is $7 \mathrm{MHz}$ total length of the site, transmit and receive part, is around $800 \mathrm{~m}$.

According to the client's requests in the phase of planning the coverage zone should be calculated in advance. The maximum coverage range depends on frequency, day/night time, sea state, radiated power... In order to achieve coverage of complete EEZ the network of the sensors with overlapped coverage zones should be used [14].

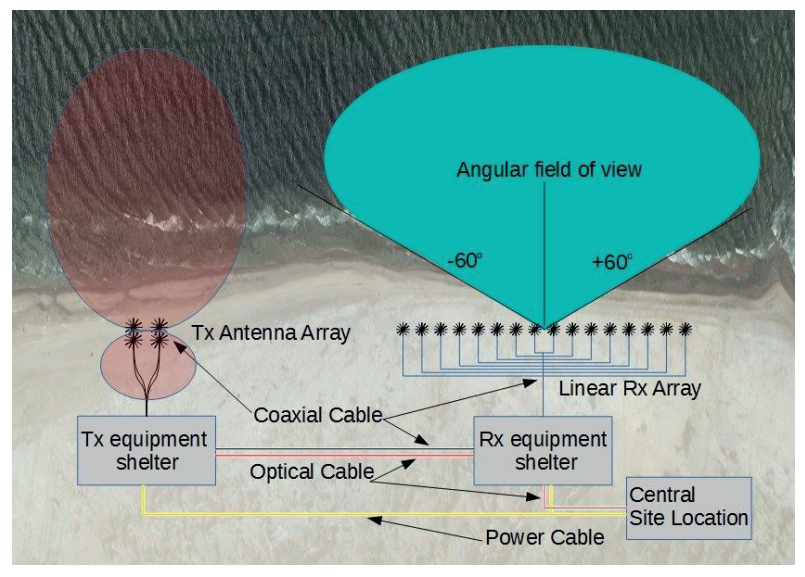

Fig. 4. Site configuration of HF-OTHR

Manual drawing polygons in the form of coverage area for all sensors for complex systems is a difficult and complicated task, so this solution provides possibility of automatically generating polygon for each sensor using the software tool.

\section{APPLICATION OF THE SOFTWARE TOOL GOOGLE EARTH FOR VISUALISATION OF MSS DESIGN}

In the early phase of the design of MSS is necessary to initially conduct an analysis of the coastline where the potential sensors will be installed. The analysis is done through one of the software tool for visualization of the Earth's surface.

Currently free on the market and the most represented version of this software tool is Google Earth (GE) [15]. Google Earth is a virtual globe, a map and geographic information software. This software maps the Earth through the superimposition of images obtained from satellite imaging, aerial photography and the Geographic Information System (GIS) 3D Globes. The file formats that can be displayed in GE are KML or KMZ.

A software tool that is used to automatically generate polygons for all above mentioned sensors is Matlab. It has built-in functions for location computing in World Geodetic System (WGS) [16]. A purpose of this solution is to automatically calculate and draw the observation zones of all sensors individually generating a unique KML file [15]. For a graphical representation of KML file software tool Google Earth (GE) is easily used.

Algorithm for design of MSS is shown on the Fig. 5. Using GE, it is possible to notice the free space near the 
coast suitable for installing the sensors. A very useful option provided by GE is usage of measuring tools by means of obtaining information about the distance between potential locations and thereby obtaining awareness of sensors arrangement and spatial distribution in order to make the best decision. After placing the placemark for the potential sites, on the basis of the previous analysis of the coast, it is necessary to export all of those potential sites with the geographical coordinates in one unique Comma-Separated Values (CSV) file. CSV is a "text-based" format. It is two dimensional structures consisting of rows of data, each row containing multiple cells [17]. Each row carries information about sensors. Cells, from each row, carry information about name of the sensors, maximum range, latitude, longitude, and true north, respectively. Fig. 6 shows an example of CSV file.

For calculating the coverage area of the HF-OTH radar is necessary to use predefined CSV file which carries information about name of the sensors, maximum range, geographic position of the receiving $(\mathrm{Rx})$ antenna array and angle relative to the north (true north), Fig. 4. A program defines a distance to the TX array, as well as a position of the TX array. After compiling the code, a program automatically draw the polygon with the coordinates of the circular sector of the angle of $120^{\circ}$ for each potential HF-OTHR site.

For calculating the coverage area of the MW radar and the EO camera is necessary to use predefined CSV file which carries information about name of the sensors, maximum range and geographic position of potential site. A program defines a distance to the HF-OTHR (if the HF-OTHR site is planned to be installed at the same area), as well as a position of the tower which carries the sensors itself. After compiling the code, a program automatically draws the polygon which presents covering area of the sensors.

Next step is to define a path of the CSV file into Matlab code. After running Matlab code, the code draws coverage areas of all sensors in the form of a polygon and generates a KML file suitable for re-display in software tool for the visualization of these polygons. The KML file specifies a set of features (place marks, images, polygons, 3D models, textual descriptions, etc.) that can be displayed on maps in geospatial software implementing the KML encoding. After loading the KML file generated by the Matlab code, GE displays the complete coverage area.

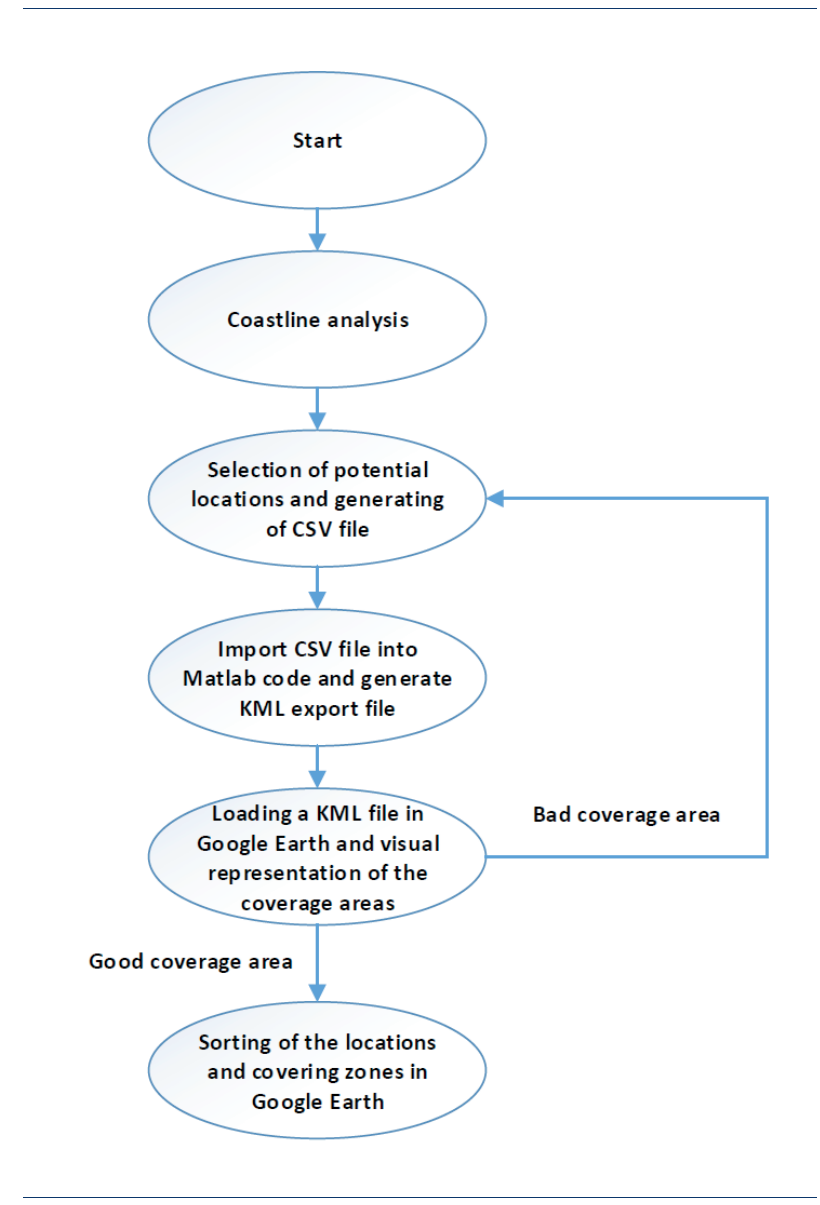

Fig. 5. Algorithm for design of MSS

\begin{tabular}{|l|r|r|r|r|}
\hline othr1 & 200 & -35.4673 & -57.1432 & 193 \\
\hline othr2 & 200 & -36.8675 & -56.6671 & 170.76 \\
\hline othr3 & 360 & -37.3801 & -57.0488 & 201.44 \\
\hline othr4 & 360 & -38.3078 & -57.8906 & 244.25 \\
\hline othr5 & 360 & -38.7874 & -59.6397 & 253.92 \\
\hline othr6 & 200 & -39.6224 & -62.1089 & 200.84 \\
\hline
\end{tabular}

Fig. 6. CSV file format

If global coverage is homogeneous, without interruptions and "holes" in the area of coverage of the EEZ, this KML file is adopted as a potential solution and goes to the next phase of the design of the solution, Fig. 5. That phase is the field survey and search for the best permanent location in the vicinity of the predefined potential location from GE.

If global coverage is not homogeneous and has interruptions and "holes" in the coverage area of the EEZ is necessary to do the rearrangement of the sensor In GE and repeat procedure from point in algorithm showed on Fig. 5-Selection of potential locations, until the covering area is fully covered by all installed sensors. 


\section{VISUAL REPRESENTATION OF THE ACHIEVED RESULTS}

The required data for calculating the archived coverage area for all sensors used in MSS are the geographical location of potential locations where sensors would be installed, coverage range and true north. Following three figures show through examples how the polygons, generated by our code in Matlab, look for each sensor individually. In the Fig. 7 a polygon generated in Mablab and displayed in GE for HF-OTHR is presented. In the Fig. 8 a polygon generated in Mablab and displayed in GE for MW radar is presented. In the Fig. 9 a polygon generated in Mablab and displayed in GE for EO system is presented.

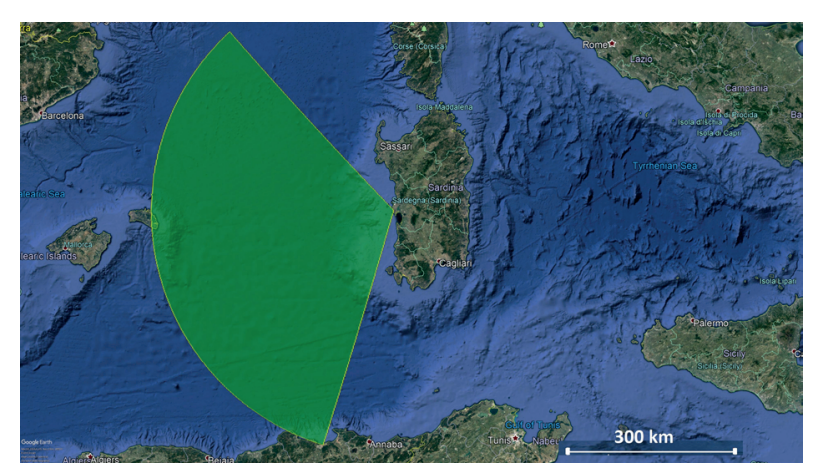

Fig. 7. HF-OTHR polygon generated in Matlab and displayed in GE

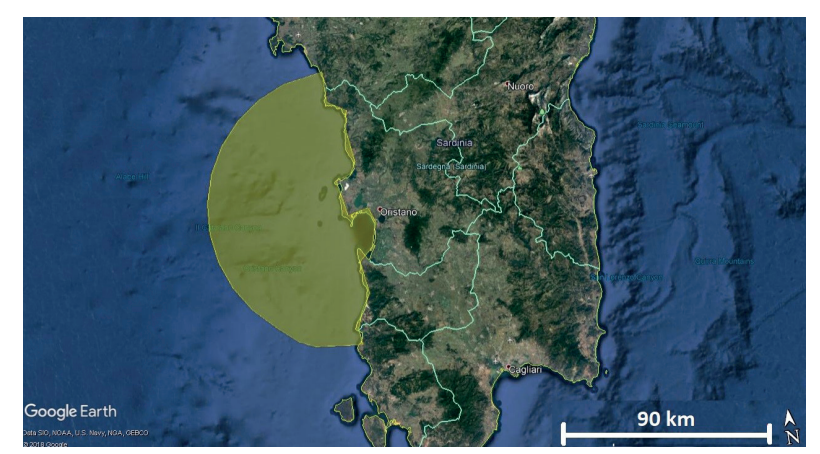

Fig. 8. MW radar polygon generated in Matlab and displayed in GE

In order to present a complete sensors network Sardinia island was used for demonstration, Fig. 10. From the Fig. 10 can be noticed all the coverage areas for the following sensors: Over the Horizon Radar (OTHR) green color, MW radar - yellow color, EO camera - blue color. Also, compleate sea area is fully covered by the sensors. The global coverage is homogeneous, without interruptions and "holes" in the area of coverage of the EEZ, so this would represent an example of successive MSS design.

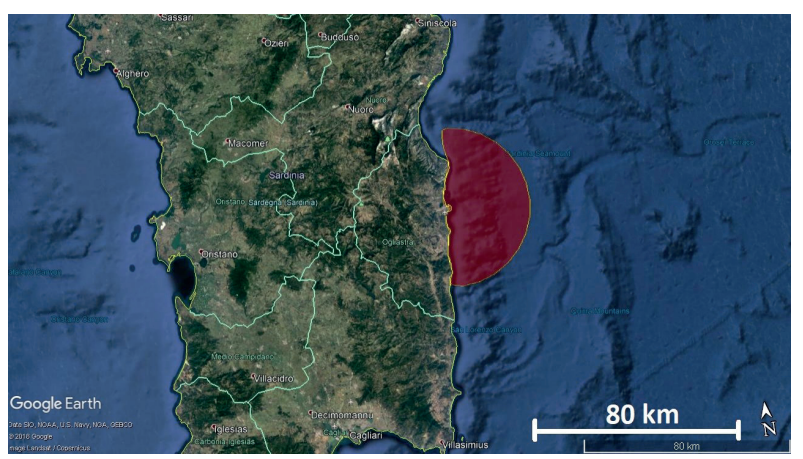

Fig. 9. EO camera polygon generated in Matlab and displayed in GE

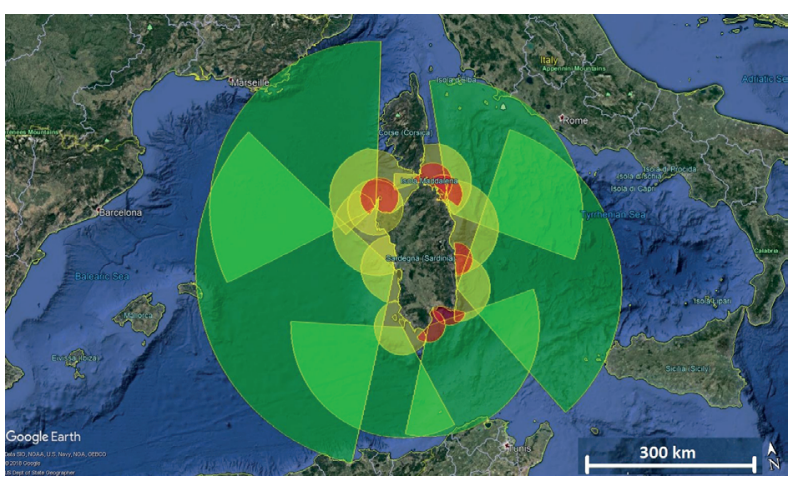

Fig. 10. Total coverage area polygons generated in Matlab and displayed in GE

In the Fig. 11 GE structure of the imported polygons and position of the sensors is presented. The structure of graphic elements is complex and includes a large number of individual polygons. Polygons are loaded in the layers, so for the analysis purpose only one type of the sensors can be displayed, or all sensors from only one site can be displayed, etc.

Easy manipulation with particular elements, editing and importing on various maps in just a few steps represents the obvious advantage of implemented solution over any manual design and hand-made manipulations over a number of objects. 


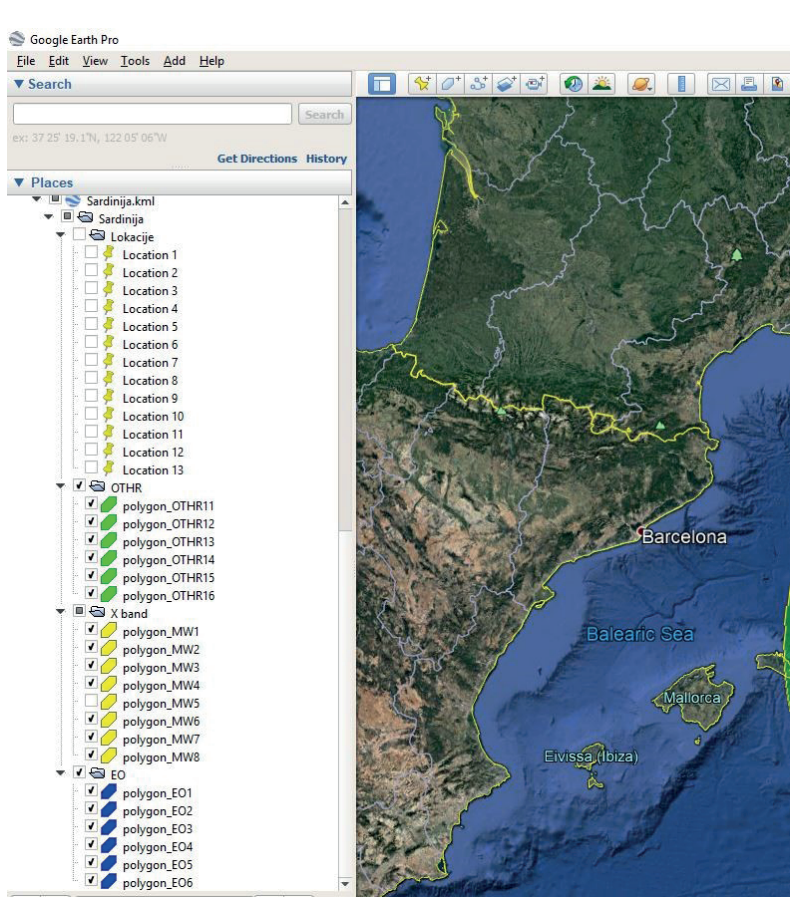

Fig. 11. Google Earth structure of the imported polygons

\section{CONCLUSIONS}

We are all witnesses to changes in the society and entangled paradigms. Over the past few decades we have seen increased threats of terrorism, smuggling, theft of national wealth, etc. With all those global events monitoring of the EEZ becomes almost mandatory. It is safe to assume that this type of MSS, which can provide monitoring of complete EEZ, will find the place in global use in the near future.

In this paper main focus is given to provide solution which is completely automated, fully reconfigurable and easy to import in software tool for the visual representation, such as GE. Taking into account all these aspects, together with careful system design of each sensor in the network leads to complete EEZ monitoring. This solution found place in practical application. It is used to make solutions for the Vlatacom Institute, also applied to already operational MSS around the world.

\section{REFERENCES}

[1] L.D.Sevgi, A.M.Ponsford and H.C.Chan, "An integrated maritime surveillance system based on highfrequency surface-wave radars, Part 1: Theoretical background and numerical simulations," IEEE Antennas and Propagation Magazine, Vol.43, no. 4, pp. 28-43, Aug. 2001.
[2] L.D.Sevgi, A.M.Ponsford and H.C.Chan, "An integrated maritime surveillance system based on highfrequency surface-wave radars, Part 2: Operational status and system performance," IEEE Antennas and Propagation Magazine, Vol.43, no.5, pp. 52-63, Oct. 2001.

[3] D. Nikolic, N. Stojkovic, N. Lekic: "Maritime Over the Horizon Sensor Integration: HFSWR and AIS Data Integration Algorithm", Sensors Magazine, Vol.18, Issue 4, April 2018.

[4] B. Dzolic, D. Nikolic, N. Tosic, N. Lekic, V. Orlic, B. Todorovic, "System for remote monitoring and control od HF-OTH radar", OTEH 2016, Belgrade, Serbia, October 2016.

[5] D. Nikolic, N. Tosic, B. Dzolic, N. Grbic, P. Petrovic, A. Djurdjevic, N. Lekic, “Tailoring OTHR Deployment in Order to Meet Conditions in Remote Equatorial Areas", CSPA 2019, Penang, Malaysia, March 2019.

[6] D. Nikolic, Z. Popovic, M. Borenovic, N. Stojkovic, V. Orlic, A. Dzvonkovskaya, B. Todorovic: "MultiRadar Multi-Target Tracking Algorithm for Maritime Surveillance at OTH Distances", IRS 2016, Krakow, PL, 11-15 may 2016.

[7] P. Petrovic, N. Grbic, B. Dzolic, N. Lekic, M. Peric, "Software for Monitoring of Direct Path Test Data for HFSW Over the Horizon Radar", IcETRAN 2018, Palic, Serbia, June 2018.

[8] "SEAFALCON-S", Product datasheet, GEM elettronica, http://www.gemrad.com/wp-content/ uploads/2017/01/SEAFALCON-S.pdf.

[9] M.I.Skolnik, "Radar Handbook, Third Edition," McGraw-Hill, 2008.

[10] "vMSIS3-CHD-1200-U - Vlatacom Multi Sensor ImagingSystem 3 - Cooled High Definition”, Product datasheet, Vlatacom Institute, https://www. vlatacominstitute.com.

[11] Electrical Characteristics Of The Surface Of The Earth, Recommendation ITU-R P.527-3, CCIR, Geneva, 1992.

[12] N. Tosic , B. Dzolic, D. Nikolic, N. Lekic, B. Todorovic, "Izazovi pri projektovanju HFSW radara”, ETRAN , Zlatibor, Srbija, Jun 2016.

[13] D. Nikolic, B. Dzolic, N. Tosic, N. Lekic, V. Orlic, B. Todorovic, "HFSW Radar Design: Tactical, Technological and Environmental Challenges", OTEH 2016, Belgrade, Serbia, October 2016., in Serbian.

[14] P.Braca, R.Groso, Jochen Horstmann, and Raffaele Grasso, "Maritime surveillance with multiple overthe-horizon HFSW radars: An overview of recent experimentation“, IEEE Aerospace and Electronic Systems Magazine,Vol.30, Issue 12, January 2016.

[15] J. Wernecke, “ The KML Handbook, Geographic Visualization of the Web", Addison-Wesley, Upper Saddle River. ISBN 978-0-321-52559-8 
[16] G. Seeber, "Satellite Geodesy" 2nd completely revised and extended edition, Walter de Gruyter, Berlin, New York 2003, ISBN 3-11-017549-5
[17] B. Girault, S. Narayanan, A. Ortega, P. Goncalves, E. Fleury, "A matlab toolbox for graph signal processing”, ICASSP, New Orleans, LA, USA, March 2017. 